\title{
Discussion on the Teaching Reform of the Adaptive Control Course for the Postgraduate Students
}

\author{
Zhou Danfeng*, Li Jie \\ College of Intelligence Science and Technology, National University of Defense Technology, Changsha, China
}

\section{Email address:}

zdfnudt@163.com (Zhou Danfeng),Jieli@nudt.edu.cn (Li Jie)

${ }^{*}$ Corresponding author

\section{To cite this article:}

Zhou Danfeng, Li Jie. Discussion on the Teaching Reform of the Adaptive Control Course for the Postgraduate Students. Science Innovation. Vol. 7, No. 2, 2019, pp. 59-63. doi: 10.11648/j.si.20190702.12

Received: April 19, 2019; Accepted: May 23, 2019; Published: June 15, 2019

\begin{abstract}
The Adaptive Control is an important course for the postgraduate students of Control Science and Engineering. However, in the teaching it is found that there exist some problems: large amount of theoretical formulas, lack of study interests for students, difficulties in learning, poor effect of study, etc. Aiming at these problems, combined with the opinions gathered from the students, this paper tries to analyze the reasons that lead to these problems, and then tries to provide some suggestions on the teaching reform of this course in four aspects, such as the contents selection of this course, the ways of teaching, etc. The attempts of seminar-style teaching in the Adaptive Control course are mainly discussed as well. This work also provides a reference for the teaching reforms of related strong theoretical post-graduate student courses.
\end{abstract}

Keywords: Adaptive Control, Teaching Method, Research, Course

\section{研究生课程《自适应控制》教学改革探讨}

周丹峰", 李杰

国防科技大学智能科学学院, 长沙, 中国

邮箱

zdfnudt $@ 163 . c o m$ (周丹峰), jieli $a$ nudt.edu.cn（李杰）

摘要: 《自适应控制》是控制科学与工程专业的一门重要的研究生课程。然而在教学过程中发现这门课程在讲授时存 在理论公式繁多、学生兴趣不高、学习难度大、学习效果差的问题。本文针对这些问题, 结合学生课后反馈的意见, 深入剖析产生这些问题的根本原因, 然后重点从教学内容、教学方法等四个方面提出对这门课程的教学改进建议, 并 重点探讨了研讨式教学在《自适应控制》课程教学中的尝试。本文可以为相关理论性较强的研究生课程改革提供借鉴。

关键词: 自适应控制, 教学方法, 研究, 课程

\section{1. 引言}

相对本科阶段的课程, 研究生专业课程在深度和难度 上都有很大提高, 需要学生有较为深厚的理论基础。《自 适应控制》课程是我校控制科学与工程专业研究生课程。
经过多年的教学实践发现, 该课程在教学过程中存在学生 学习兴趣低、学习难度大、学习效果欠佳的问题。由于课 程内容抽象, 理论公式繁多, 讲授难度大, 如果处理不好 容易出现“讲不清、学不明、用不上”的情况[1]。而这些问 题也是理论性较强的研究生课程普遍存在的问题。如果无 法及时解决上述问题, 则类似《自适应控制》这样的理论 
性较强的课程将沦为学生谈之色变的高难度课程, 教学效 果将大大折扣。

这一问题在高校课堂中普遍存在, 已经引起众多学者 和教育工作者的重视。在教学方法上, 案例教学、启发式 教学是被普遍认可的一种教学模式。针对理论性较强的研 究生课程实际情况, 有的学者提出了“理论-案例-讨论”相 结合的教学方法, 用工程案例引导学生参与讨论, 从而调 动学生积极思考问题[2]。这种方法在工程性背景较明确的 课程中是值得推广实践的。启发式教学是近年来教学实践 中被广泛推荐的一种方式, 这种方法以问题为导向, 能够 调动学生学习的主动性和积极性。陈善勇等在研究生课程 《数字化制造技术》的教学改革中尝试了启发式教学, 取 得了不错的效果 [3]。有的学者针对《系统辨识与自适应控 制》课程的教学过程出现的学生乏味、厌学的问题, 主张 改变“老师讲、学生听”的传统教学方式, 从教学内容、教 学手段、考核方式等方面提出了各自的建议 [4,5]。翻转课 堂也是近年来备受关注的一种创新教学模式。针对《自动 控制原理》课程的教学改革, 陶洪峰提出开展翻转课堂与 引导式教学相结合的教学方法, 增加学生学习的自由度, 改变单纯由老师授课、学生听讲的传统课堂形式[6]。

在课堂形式和考核形式上, 有的学者进行了大胆的创 新尝试。针对研究生阶段信号处理课程理论性较强的问题, 有的学者主张将最新的现代信号处理技术和工程应用引 入课堂, 而非单纯讲授理论知识, 并结合授课和考核方式 的改革, 在研究生培养上取得了丰硕的成果[7]。此外, 在 研究生理论课程教学中, 有的学者将课堂理论教学、学术 论文研究和科研项目攻关三者联系起来, 构成三位一体的 立体式教学, 设置以课程学术论文为核心的考核标准, 要 求学生把课堂内容结合科研实际撰写发表DEVS学术创新 课程学术论文, 其考核比例达到 $50 \%$ 。这就迫使学生在有 限的时间内把所学内容和工程结合起来并发表学术论文 [8]。这种方法有一定的借鉴意义，但其通用性值得思考。

研究生课程的另外一个特点是内容复杂, 但课时数少, 此时对教学内容的取舍必须深入考虑。例如, 针对36课时 的《自动控制原理》课程, 学生学习难度大、兴趣低, 有 学者在教学改革中主张更新课程理念、大力简化教学内容 [9]。

此外, 有的学者从研究生教育管理的角度, 分别以研 究生的科研、创新能力方面着手, 对研究生课程的改革建 设提出了改革意见, 主张在课程设置、内容设置方面慎重 考虑, 并革新教学模式, 以学术研究为牵引, 采取多元、 灵活的课堂形式, 以课堂教学提高研究生的科研、创新能 力 $[10,11]$ 。

总体来说, 在研究生教学改革中, 要扭转学生对理论 性较强的课程产生的枯燥、乏味的印象, 一方面需要在教 学内容上进行适当的取舍, 另一方面以问题为导向的启发 式教学、以及将工程应用引入课堂的案例式教学方法是值 得大力推广的。本文针对《自适应控制》研究生课程讲授 过程中出现的问题, 结合学生反馈的意见, 首先对这些问 题出现的深层次原因进行剖析, 然后结合课堂实践, 提出 有针对性的改进方法, 尤其是对《自适应控制》研讨式课 堂的可行性进行了探讨, 为理论性较强的研究生课程教学 改革提供参考。

\section{2. 《自适应控制》教学过程中存在的问题}

自适应控制是介于现代控制理论和智能控制理论之 间的一种高级控制理论。在实际控制工程中, 被控对象和 环境都比较复杂, 而且在运行过程中系统参数还会发生变 化, 难以建立精确的数学模型, 此时便需要用到自适应控 制的方法。自适应控制在发展之初就是为了解决工程控制 问题而发展起来的, 因此是一门实用的课程。然而这门课 程需要较深厚的理论基础, 繁多的公式往往让很多学生感 到困惑, 反而认为自适应控制是一门不实用的理论课。下 面对该课程教学过程中出现的主要问题进行列举, 同时剖 析出现这些问题的原因。

\section{1. 学生选课积极性不高}

《自适应控制》是我校研究生的一门选修课, 同时面 向硕士研究生和博士研究生开放选修。然而根据近7年的 选课情况来看, 学生选课的积极性远不及其它工程性或实 践性课程高。这主要是因为研究生阶段的理论性课程通常 具有一定的理论深度, 往往比较抽象, 学习难度较大。而 《自适应控制》课程最大的特点是公式多、内容抽象, 学 生学习兴趣不高。其次是长期以来学生们对这门课程形成 了一定的偏见, 通过往届学生的描述, 就形成了对这门课 程的固有印象, 甚至谈之色变, 因而不敢选。

要打破学生这种固有成见, 提高学生选课积极性, 只 有从授课内容、授课方式上作出本质的调整, 使得学生能 够真正理解、掌握课程的主要内容, 才能改变学生的固有 看法, 逐渐提高学生的选课积极性。

\section{2. 教学内容容易使学生产生枯燥感}

自适应控制是一门理论性较强的课程, 课程内容不可 避免出现较多的数学公式。这对于大多数学生来说, 课堂 的内容会显得枯燥乏味。本课程在课程结束时, 都会要求 学生对本课程的课堂教学及各自的学习情况用书面的形 式进行反馈。根据反馈的情况来看, 至少一半的学生在反 馈的意见中都提到了课程内容公式繁多、枯燥的问题。这 说明这一问题具有一定的普遍性。

究其原因, 无外乎以下几点: (1) 授课内容对学生 的授课功底要求较高。无论是模型参考自适应还是自校 正控制, 都离不开对控制模型的描述和控制律的推导, 这中间往往涉及很多数学公式。例如, 自校正控制部分 如果加入辨识环节后, 其数学表达式往往较长, 这种表 达式会对学生产生强烈的视觉冲击, 给人一种十分复杂 的感觉; 而如果写成简化的表达式, 学生又无法认清公 式的具体结构, 在形式上过于抽象而不易理解公式的含 义; (2) 授课内容的取舍不合理。自适应控制的很多定 理是有严格的数学证明的, 然而要在有限的课时数内把 这些证明都讲授清楚就需要列举大量的公式, 从而使学 生感到乏味。例如, 前几年在讲解最小方差调节器时, 在课堂上证明了带自校正算法的最小方差调节律能够 收玫到理想的最小方差调节律, 这一过程需要大量的矩 阵表达式、求和表达式、互相关和自相关推导、过程相 当复杂, 花费了很多课堂时间。然而此举却起到了适得 
其反的效果, 学生不仅很难弄明白推导的逻辑, 而且使 得大部分学生失去学习兴趣后开始分神。试想这样的内 容怎会引起学生的兴趣。研究生普遍已经具有相当强的 自学能力, 类似这样枯燥的推导过程完全可以删减或留 给学生课下自己推导。(3) 没有把数学公式对应的物理 意义讲明白, 使学生陷入公式的泥沼而无法从总体上把 握整体思路。不少学生在反馈的意见中提到课程公式太 多, 对公式的物理意义难以把握。这也是造成授课过程 枯燥乏味的重要原因。研究生阶段的理论课程应重点讲 解解决问题的总体思路、控制律产生的基本原理。即使 在推导的过程中也应不忘介绍公式在整个过程中的逻 辑关系。例如, 在讲解各种自校正算法的在线辨识问题 时, 圥长的辨识公式往往会给人一种过于复杂的感觉, 然而如果把这些公式里不同类型的项进行归类, 并将它 们和实际辨识对象的结构联系起来, 配合形象的图形或 动画描述, 则学生会更容易理解。

\section{3. 教学形式不当容易引起学生接受困难}

好的教学形式能够增进学生对抽象知识的理解。然 而对于理论性较强的课程, 做到这一点并不容易。我校 的《自适应控制》课程在早些年侧重板书推导, 由教员 在黑板上以手写的形式进行公式推导和讲解。其优点是 可以以清楚的形式展现公式的来龙去脉。但其最大的问 题是当公式较多时, 教员需要不停地在黑板上进行板书, 而学生则需要不停地做笔记, 很难有足够的时间对抄录 的内容进行消化领会, 更无法把握授课内容的整体思路。 此外, 板书需要消耗较多的时间, 这意味着对于有些问 题无法深入展开讨论, 这在一定程度上增加了学生理解 的难度。

为了克服这些问题, 在随后的教学中我们开始采用 多媒体授课方式, 课堂教学主要以老师讲授配合多媒体 课件展示的形式。这种方式在一定程度上克服了纯粹板 书方式的限制, 但是在教学中发现这种方式同样存在不 少问题。首先, 多媒体课件是事先制作好的, 诸多复杂 的公式往往是在很短时间内就完全展示出来, 过快的节 奏通常也难以取得良好的接受效果。实际上, 试图用多 媒体课件在短时间内展示大量的公式反而会引起学生 注意力的下降, 使得讲授效果变差。其次, 多媒体课件 每页展示的内容是有限的, 当推导过程比较复杂时, 课 件的频繁换页会导致推导过程的前后连贯性变差。这个 问题对于逻辑性较强的理论性课程来说是比较严重的, 因为学生无法参考前面的某些结论或公式。此外, 如果 多媒体课件在展示效果上显得呆板而缺乏变化, 学生很 快会感到视觉疲劳、走神, 这也是过多依赖多媒体课件 所带来的问题。

\section{3. 教学改革探讨}

针对以上列举问题, 这些问题也是很多理论性较强的 研究生课程面临的共性问题。下面结合《自适应控制》的 实际情况, 对此类研究生课程教学提出如下改进建议。

\section{1. 大力改革教学内容}

自适应控制是上个世纪六、七十年代发展起来的控制 方法, 到目前为止已经产生很多改进的自适应控制方法, 自适应控制的文献也浩如烟海。然而因课时有限, 以往在 课堂上讲授的内容多以基本的模型参考自适应控制和自 校正控制为主。然而, 考虑到研究生阶段的学生已经具有 较强的自学能力, 因此课堂教学内容应避免完全按照教材 上的内容照搬照抄。在教学实践中发现, 过于强调对基础 性结论的证明和推导往往会得到适得其反的效果。例如, 自校正控制的基础是系统辨识, 然而我校研究生学生的层 次是不同的, 因此在课堂上仍会适当加入系统辨识的相关 讲解。但是在讲解递推最小二乘辨识时如果花费大量时间 从矩阵求逆引理开始证明, 则有很大的概率会使学生丧失 学习兴趣、产生疲劳。实际上, 课堂上只要给出递推最小 二乘的公式并解释每个公式的意义即可, 对其推导过程则 可以大幅删减。

随着自适应控制、智能控制的发展, 自适应控制已经 发展出很多分枝, 有的分枝与其它学科相互交叉。因此在 课堂上, 应当对教学内容进行大刀阔斧的调整, 适当压缩 一些复杂的推导过程, 加入自适应控制的最新研究成果, 以开阔学生的眼界。但是这类知识宜点到为止, 否则会使 得教学内容杂乱无章而失去主线。

\section{2. 采用更加生动的教学方式}

前已叙及, 采用多媒体课件授课的方式有助于提高教 学效率。然而, 即使像《自适应控制》这类理论性较强的 课程, 也不应忽视多媒体课件的视觉效果。综合采用层次 分明、动静结合的展示方式能起到突出重点、吸引学生注 意力的作用。例如, 在讲解Diophantine方程的解法时, 可 以利用案例把Diophantine方程的求解过程用动画的形式 制作出来, 然后借助遥控激光笔的控制, 一步一步展示给 学生, 使得学生在课堂上即可轻松掌握这种求解方法。从 课后的考核情况来看, 多半学生都能能够按照课堂上介绍 的方法编写程序进行求解。又如, 在讲解自校正PID控制 时, 我们首先在多媒体课件上用动画演示磁悬浮系统的工 作原理（如图1所示），并在此基础上讲解系统建模、离 散化方法, 然后用自校正PID对磁悬浮系统进行控制仿真。 学生对该案例十分感兴趣, 课后仍有不少学生主动同授课 教师探讨相关问题, 这同之前的教学效果形成了鲜明的对 比。

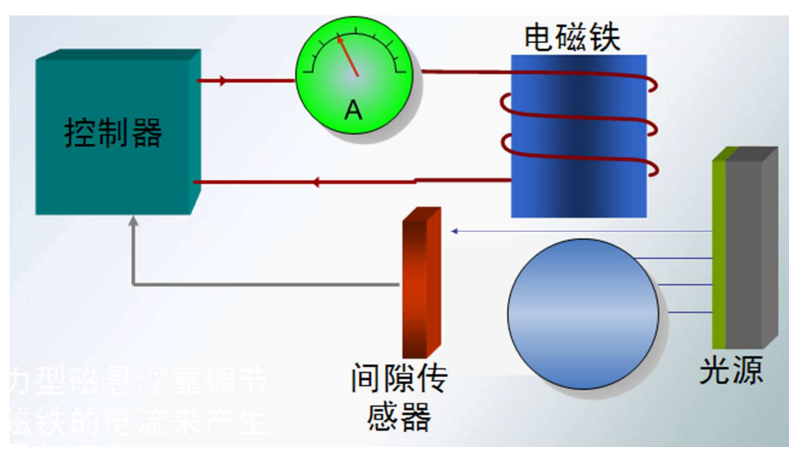

图1 讲解自校正PID时用到的磁悬浮系统原理演示动画。 
另外，考虑到《自适应控制》课程在讲解时不可避免 会出现大量公式, 此时不建议将这些公式一次性投射在屏 幕上。实践表明, 采用逐步推进、根据进度逐步展示的形 式能够使学生更好地把握推导过程的来龙去脉。在幻灯片 切换时, 可以保留上一页幻灯片的部分重要结论、公式、 图片, 在切换进入下一页时可以利用动画把它们移动到新 的位置上, 这样既不影响新幻灯片的展示, 又能起到参考 作用。根据学生课后的反馈情况以及课堂上听课教师的反 馈意见来看, 采用动画+逐步推进+带过渡的幻灯片切换方 式十分有助于讲解公式繁多的理论性课程。

此外, 采用多媒体教学的形式并不意味着放弃板书方 式。实际上, 将二者有效结合方能起到最佳效果。对于一 些需要在后续推导中用到的公式或结论, 可以将它们写在 黑板上, 以便于学生参考。

\section{3. 注重案例教学和启发式教学}

近年来, 案例教学、项目教学法在有的课程教学改革 中得到了充分重视[12]。在《自适应控制》的教学过程中, 我们有意将一些工程上的案例带入课堂, 有针对性地对这 些案例进行讲解, 收到了很好的效果。例如, 在讲解系统 辨识时, 我们以磁悬浮系统为例, 特意在磁悬浮列车的转 向架上进行了悬浮试验, 并对悬浮系统施加特定的扰动, 然后对悬浮间隙、电流进行测量, 利用系统辨识的方法来 估计悬浮系统的参数。这一案例在课堂讲解时, 学生兴趣 较高, 现场提出了很多问题。又如, 在讲预测控制时, 我 们将火星车的通信和控制案例带入课堂, 使得学生很自然 地领会到预测控制的基本思想, 比单纯从公式上进行讲解 更加生动形象。案例教学的方法既开阔了学生的眼界, 又 能很好地把课堂的知识融入其中, 学生反响较好。

启发式教学是近年来教育教学工作中大力推广的一 种方式, 通过设置合理的问题或案例来启发学生思考, 进 而引入课堂知识的讲授。这一方法对于理论性较强的课程 具有很好的应用效果。例如, 在讲述最小二乘辨识原理时, 我们首先给出一组电磁铁在电流作用下铁芯温度随时间 变化的离散测量点, 让大家判断电磁铁的温度变化符合什 么样的规律。当学生从直观上得出抛物线或者指数曲线的 规律后, 又问学生如何确定一条最优的抛物线或指数曲线 来逼近电磁铁真实的温度变化曲线, 这样很自然地引导学 生进行思考, 进而自然地引出最小二乘拟合的基本原理。 这种方法可以使学生对最小二乘的基本原理产生深刻的 认识。

\section{4. 尝试研讨式教学}

研讨式教学采用课堂上学生和老师、学生和学生之间 针对某些特定问题开展讨论的方式来体现教学内容。这种 教学方式早已有之, 但将其应用于理论性较强的课程中尚 不多见。近年来, 我们尝试在课堂上安排一定时长的研讨 环节, 研讨的内容大部分来自前一次课堂上点到但并未展 开细讲的内容、或者是与课程知识相关的某些扩展性内容。 这些研讨的内容通常需要学生在课下查阅一定量的文献, 并经过自己的思考和加工。研讨的形式为 $3 \sim 5$ 人一组, 小 组成员自行商讨分工, 在课前针对某一个或两个特定的问
题收集资料, 开展研究, 然后进行总结。在课堂上指定一 位学生在讲台上进行讲解。在讨论环节, 所有的学生都需 参与讨论。这种形式相对以往单纯由教师在讲台上讲授的 方式而言有较大的改进, 大部分学生对这种形式的研讨方 式持肯定态度, 认为这种方式一方面可以通过研讨、查阅 资料等方式对所研究的问题有深刻的认识, 另一方面也可 以使每个人都得到锻炼。

然而通过最近两年的教学实践来看, 这种方式有利也 有弊。总体来看, 研讨的内容要想激发所有学生都参与讨 论需要具备两个条件: 第一, 所有参与者对讨论的内容均 有一定的了解; 第二, 所有参与者对讨论的内容都感兴趣。 但是对于理论性较强的内容来说, 要具备这两点并不容易。 例如, 有的学生在反馈的意见中提到这种方式对于准备研 讨的小组成员来说收获更大, 但是不能保证所有学生都对 所提的问题感兴趣, 这样会导致课堂上没有事先准备的同 学难以参与讨论, 从而使研讨陷入尴尤的局面。在这种情 况下, 教师对于研讨课堂的把控能力就显得格外重要。话 题的引入、背景介绍、问题的引出、现场的主持能力、时 间的把控等方面都很重要。最关键的是, 教师要对讨论的 议题做好充分的准备, 并准备好在讨论中可能引出的问题 及参考答案。

总之, 对于理论性较强的课程来说, 研讨课堂是一件 值得探索、实践的教学改革模式。但是从近两年的实践经 验来看, 这类研讨型课堂在整个课程中的比例不宜过高, 否则会使得整个课程的教学内容过于发散而出现知识碎 片化的问题。

\section{4. 结论}

《自适应控制》是控制科学与工程专业研究生课程中 比较重要的一门专业课程, 它来源于控制实践, 但是具有 一定的理论深度, 学习难度较大。如何改革这门课程的授 课方法, 改变以往“讲不清、学不明、用不上”的状况, 引 导学生对这门课的知识进行深入理解和消化是需要重点 研究的内容。本文根据近年来教学实践中发现的问题以及 学生对这门课程的反馈意见, 剖析产生问题的根本原因, 重点从四个方面提出对应的改革措施: 教学内容、教学方 式、案例教学、研讨式教学。尤其对我校《自适应控制》 课程近两年的研讨式教学尝试进行了分析, 认为这种方式 可以作为理论性较强的课程的一种探索, 但是它对教师的 课堂组织、知识结构、课堂把控能力要求较高, 其实际效 果需要在未来的实践中进行进一步检验。

总之, 只有在教学实践中对《自适应控制》课程的教 学内容和教学方法进行大刀阔斧的改革, 才能从根本上扭 转学生对这门课的固有成见, 提高学生的学习兴趣, 使学 生真正掌握这门课程的精髓。

\section{致谢}

本文为国家十三五重点研发计划子任务《中速磁浮列 车车轨耦合振动抑制方法研究》(2016YFB1200601-B12) 的阶段性成果之一。 


\section{参考文献}

[1] 胡永江,王永川,马晓琳.军校研究生“数字信号处理”课程改 革 $[J]$.电气电子教学学报,2015,37(3):45-47。

[2] 孔庆军. 基于激发研究生的学习动力与兴趣的课程改革研 究[J].教育教学论坛,2018,43:130-131。

[3] 陈善勇,戴一帆,彭小强, 解旭辉.“回归工程”背景下的《数字 化制造技术》研究生课程改革探索 [J]. 高等教育研究学 报,2009,第32卷增刊:37-39,45。

[4] 王坤. 《系统辨识与自适应控制》课程教学过程研究 [J]. 教 育教学论坛, 2017,14:167-168。

[5] 王建国.《系统辨识与自适应控制》课程教学改革探讨 [J]. 科 技信息，2010,36:11-11。

[6] 陶洪峰.自动控制原理课程的混合式教学模式探索 [J]. 大学 教育, 2019.3:62-64。

[7] 刘志刚,赵舵. 研究生信号处理课程改革研究 [J]. 电气电子教 学学报,2011,33(2):32-33。

[8] 李小波,王涛,雷永林,朱一凡,王维平.学术创新导向的研究 生理论课程教学方法研究——“建模仿真理论”课程为例 [J].高等教育研究学报,2018,41(4):111-116。

[9] 石建平, 刘鹏.新工科背景下自动控制原理课程教学改革[J]. 教育教学论坛,2019,8:138-139。

[10] 王鹏,张宝华.面向科研能力提升的研究生教育课程改革 [J]. 中国现代教育装备,2018,299:25-26,42。

[11] 葛治波.提高研究生科研、创新能力的切入点: 抓好研究生 课程教学 [J].扬州大学学报(高教研究版),2005,9(4):66-68。

[12] 姚秀娟,薛坐远.项目教学法在高校《可编程控制技术》课程 教学中的应用研究[J].高教学刊,2019,3:97-99。 